资源的投入，降低了运算难度，同时也提高了运算的精确性 和稳定性。其次，BIM 技术是利用信息三维模型所产生的数 据库，从该数据库中可以实现对整体施工全过程的周期信息 的存储, 包括建筑的设计阶段、建筑的具体施工过程以及完 工之后的验收阶段。另外, BIM 数通过开放的数据标准可以 实现建筑行业相关系统之间的有效的数据交换以及建筑物全 生命周期的数据管理，能够有效地支持协同工作，极大地提 高了 BIM 技术的应用效率和应用价值。随着科学技术的不断 发展以及信息时代的全面到来，人们对建筑工程项目的效率 以及建设质量的要求越来越高。[2]

当前, BIM 技术逐渐朝向全周期、深层次、系统化的方 向发展，未来也必然会应用到全生命周期的建筑系统当中。 中国将建筑全周期生命阶段规划为有效划分为策划与规划、 勘察与设计、监理与施工、运行与维护、改造拆除以及加固, 既有建筑的检测加固直接关系着中国建筑的使用寿命及使用 价值，并可以将其丁序划分为建筑生命全周期的第五个阶段。

从目前来看, 中国 BIM 技术的应用研究主要集中于新建工程 项目的发展阶段和施工阶段，对于既有建筑的检测和升级以 及加固设计方面的研究, 仍然存在严重的不足。 ${ }^{[3]} \mathrm{BIM}$ 技术是 随着智能化技术以及计算机技术而广泛发展起来的，属于最 近几年来才可以大力应用和推广的技术, 所以一些建筑年代 比较早和设计方案成型较早的既有建筑，通常不涉及 BIM 技 术的优化体系。这些既有建筑在运行和实际使用的过程中也 缺乏相应的 BIM 技术进行数据的提供与支持, 随着中国建筑 行业的不断发展以及智慧城市建设进程的制定，加快建立起 符合实际情况的既有建筑 BIM 信息模型，结合现场检测结果 进行科学的 BIM 技术应用是当前必不可少的内容之一。其次， 既有建筑在使用过程中改造和加固设计与施工、新建工程的 设计与施工以及不同种类项目施工之间存在较大的差异，这 是构成条件的不同所导致的。既有建筑的改造加固必须要明 确既有建筑的现实情况，包括既有建筑的损伤情况、结构构 件的实际材料强度、实际空间位置以及力学性能等相关因素, 而且, 既有建筑通常来说位于城市的中心, 还需要考虑在既 有建筑改造和施工的过程中要尽可能的减少对周围环境的影 响以及周围居民的干扰，选择正确的施工场地以及合理的施 工规划，保证施工项目能够顺利稳定的进行。另外，既有建 筑改造加固项目通常存在施工协同以及施工设计方面的困难,
要结合具体的施工情况进行有效的调整, 并利用改造加工施 工的相关策略进行建筑物的升级与改善, 在具体的改造加固 施工当中, 可能会涉及到各种粘贴碳纤维、焊接、粘钢、屈 曲支撑以及阻尼器的很多新型工艺和技术的应用, 现场的管 理相对比较复杂, 具有一定的管理难度。 ${ }^{[4]} \mathrm{BIM}$ 技术在既有建 筑升级改造过程中的应用目前来说主要针对于规模较大的既 有建筑, 通常体量超过 1000 平米的建筑的检测加固能够更好 地发挥 BIM 技术的应用价值。利用 BIM 技术的有效应用, 实 现既有建筑检测、鉴定、改造、设计以及施工的一体化发展， 可以为改造加工项目实现精细化的检测, 提高检测效率检测 价值, 提供符合实际情况的 BIM 模型, 使得设计方案能够得 到进一步的优化, 有效节约施工成本, 缩短施工工期, 减少 后期维护的难度, 使得运营管理更加科学和稳定。[5]

\section{BIM 技术在既有建筑改造中的有效应用}

\section{1 在项目规划与设计阶段的应用}

既有建筑改造的一个显著的特点体现在改造与升级的过 程中, 会受到多种因素的制约, 比如建筑周边的人员以及建 筑周边的环境。项目的改造效果与改造周期相对来说比较难 以得到量化和确定, 为既有建筑建立起系统科学的 BIM 模型, 利用 BIM 模型的模拟仿真功能特点以及可视化技术特点可以 为工程项目改造方案的设计与确定提供良好的条件。不同类 型房屋检测的工作重点虽然存在不同的特征点, 但是基本都 包括建筑结构完善, 测绘材料性质、倾斜以及承载力计算等 相关方面的内容。利用 BIM 模型技术呈现的报告的表现形 式可以通过照片、表格、文字说明以及图纸等多种组合形式 进行呈现, 常规的检测报告存在视觉效果差, 抽象图纸模型 信息割裂以及图纸信息的变革无法实现集中化管理等, 相关 BIM 技术在项目投资阶段与决策阶段的有效应用可以为工程 项目的具体改造情况提供源源不断的科学的数据支持。 ${ }^{[6]}$ 从 而可以利用 BIM 技术进行建筑物的整体改造, 同时, 可以对 成本进行控制, 包括项目所在地区的人力价格以及基本建材, 利用模型输出完成的单方造价信息从而可以实现对改造项目 的资金额的良好的规划，高效准确的判断资金的流出对象， 为项目的具体决策提供有效的数据支持。BIM 技术数据信息 模型为该专业的协同设计活动提供了数据集成的有效平台, $\mathrm{BIM}$ 数据共享平台可以在建筑内部进行科学的管线位置以及 
机械设备的布置。专业设计人员可以利用软件的模拟规划的 功能准确的测量建筑结构与各个位置的尺寸, 方便设计人员 及时有效地对图纸进行改造和修改, 保证图纸的质量能够满 足具体的改造要求。 ${ }^{\left[{ }^{2}\right]} \mathrm{BIM}$ 技术在项目规划阶段的应用情况与 建筑的改造规模有关, 如果改造的建筑规模相对较小, 则可 以将与既有建筑改造各专业协同设计的相关数据信息整合到 文件夹当中，并将之安放于平台，使得参与建筑改造的各个 人员都可以从服务器平台中下载相关的文件信息。同时，还 可以利用文件服平台的共享功能以及更新功能, 及时对建筑 改造的信息进行掌握和优化，使得建筑方案能够更加适用于 当前既有建筑改造的要求和环境, 实现数据资源的有效共享。 其次, 文件夹中还有各自单独的工作, 可以有针对性的开放 各个工作及比如水管改造设计, 工作人员可以查看有关建筑 结构方面的内容, 但是并不能对文件进行修改。其次, BIM 技术的可视化功能极大地提高了团队合作的效率，使得专业 的设计师之间的沟通更加的快速。基于 BIM 技术的数据模型 也可以使得各个分包团队、承包团队以及客户与供应商之间 能够迅速和准确地了解相关建筑项目的信息，减少技术交接 的时间和信息讨论的时间，并明确各方的责任，做好各责任 的划分。BIM 技术的可视化技术还可以使得各专业人员能够 更加详细的了解建筑物的构造和布局, 进行良好的设计, 明 确建筑物的空间位置信息以及建筑物的构造信息, 为建筑物 的顺利开展提供科学的数据和指导。 ${ }^{[8]}$

\subsection{BIM 技术在既有建筑检测加固鉴定阶段的应用}

既有建筑检测一般包括要准确科学的对房屋进行结构平 面、立面布置的现场测绘以及对材料的现场检测，同时还包 括对房屋沉降情况的标点以及测量和对房屋损伤情况的统计 调查并标定记录。测绘的数据内容通常包含图纸文字的形式, 在检测当中呈现原有的既有建筑检测工作持续时间较长, 所 获得的数据内容较多, 而且并不能直观地反映建筑物的实际 情况, 为后续改造和升级的施工人员的工作带来较大的难度。 后期改造加固设计人员还需要进一步全面的调查访问结构, 无形中增加了工作量, 提高了工作成本。将所有有关既有建 筑检测的信息以便按模型的形式进行呈现, 能够详细直观的 为工作人员提供数据信息，同时也可以直接使用于后续的改 造加固设计当中。 ${ }^{[9]} \mathrm{BIM}$ 模型的应用还可以融入到智慧城市管 理平台当中, 也可以作为既有建筑检测成果的, 以附加值进
行使用, 用于自由建筑的后期维护、运营维护, 对于一些平 面立面布置相对比较简单明晰的建筑来说, 可以结合现场检 测的情况以及原有的实际规划的图纸直接建立起科学的 BIM 模型。对于一些测绘相对比较困难以及原始图纸丢失的项目, 可以根据现场检测情况以及利用专业的 3D 扫描仪等相关设备 对既有建筑的点云图像进行扫描。识别既有建筑的结构变形、 空间分布、维护构件的开裂损伤等具体的情况，从而构成良 好的三维一体化模型，将数据有效的整合进入 BIM 模型当中。

\subsection{BIM 技术在项目施工阶段的应用}

BIM 系统模型包括了与建筑项目有关的所有的数据信息, 而且可以将所有的信息有效的结合到一起从而实现对建筑工 程项目地有效地集成管理。对于既有建筑检测加固项目来说, 现场的生活环境相对比较复杂，利用 BIM 技术的可视化模拟 准确地模拟施工现场的实际情况, 并利用 BIM 技术分别标注 改进项目的原有部分、新建部分以及拆除的部分, 利用其他 的造价软件以及工程量计算软件对相关数据信息进行统计计 算, 为后续工程项目的顺利建设以及有效运营提供数据支持, $\mathrm{BIM}$ 技术的在施工阶段的合理应用还可以为方案的设计工作 提供便捷的数据支持, 利用建筑模型对既有建筑围护结构的 材质、物理属性、空调通风系统方案以及组合厚度等进行更改, 从而能够更好的优化能耗评估方案。降低改造的难度, 提高 改造的效率。既有建筑改造的一个最重要的阶段, 项目施工 阶段的效率以及项目施工阶段的质量直接关系着整体改造的 效果。既有建筑相对来说存在着比较复杂的社会环境在改造 过程中, 必须要严格的进行工程资源成本的把控以及工期的 限制。在施工方法与施工工艺的要求上更加严格, 传统的项 目管理经验通常难以满足比当前既有建筑改造的需求。而且, 由于每个既有建筑改造的项目的实际情况不同, 依靠传统项 目管理经验编制的施工进度计划, 会在实际施工过程中存在 较大的波动和差异。随着进度的不同阶段、资源与成本的进 行投入也有所区别, 这导致实际的施工进度与施工设计计划 存在较大的差距, 影响施工过程中对施工计划成本以及实际 成本的良好把握。施工阶段是 BIM 技术应用的主要阶段, 也 是未来研究开发 BIM 技术的主要应用阶段, BIM 技术在施工 阶段的应用, 可以将施工过程通过 BIM 建模进行三维可视化 的展示, 从而为工作人员提供更加直观和快速的角度进行改 造设计方案的理解与遵循。同时也可以及时发现施工过程中 
出现的问题，利用模型进行设计，与施工方的有效沟通保证 施工环节能够严格按照工程项目建设标准以及内容来开展。 在施工过程中，可以利用 3D 建模系统对机电管线综合排布结 构的构建以及相关建设单位碰撞的检查和分析。

\subsection{BIM 技术在项目运营维护方面的有效应用}

既有建筑在改造完成之后，是否能够实现建筑节能、高 效、绿色、稳定的运行, 是评判企业改造效果的重要指标。 既有建筑改造通常情况下是基于 BIM 模型进行规划设计方案 的比较和确定的设计阶段的建模开发, 以及在施工阶段模型 的变革和深化，实现了既有建筑改造的大部分内容。针对既 有建筑运营维护介段的 BIM 技术的应用研究，主要针对的是 对改造过程中供暖系统设备、空调系统设备、照明系统设备 及可再生能源系统设备的管理与监控应用。BIM 技术在项目 地运营维护过程中能够集成和分析所有项目设备的运行数据, 并快速查阅设备的关键参数以及重量的技术信息, 包括设备 的运行维护情况、联系方式、生产厂商以及运行工况等, 对 于大型办公室主义及商场建筑来说, 既有建筑改造升级之后, 是否能够实现建筑的节能运行以及高效的运转是判断改造效 果的关键, 根据 BIM 模型相关数据可以建立出良好的能效管 理系统。从而可以对建筑内部各个单元用电情况进行良好的 监督与管理, 控制能源资源的消耗, 实现建筑物的绿色运行 与管理，尽可能的减少建筑系统的资源能源浪费，实现能源 用量良好的统计与反馈。BIM 模型在旧建筑改造过程中的应 用本身具有信息化的特征，业主在建筑物的实际使用的过程 中可以利用 BIM 模型将与建筑相关的基本信息进行直观可靠 的观察，包括建筑面积、各个单元的位置以及管线的分布等。

\section{4 结语}

综上所述, 本文主要针对 BIM 技术在既有建筑检测加固 中的应用进行探索，指出 BIM 的相关特点以及 BIM 技术在既 有建筑检测加固中的应用需求和具体的应用方式, 希望能够 充分发挥 BIM 技术在既有建筑检测加固改造中的作用和价值, 进一步提升既有建筑改造的效率和改造的质量。

\section{参考文献}

[1] 蒋璐, 关昊 . BIM 技术在既有建筑检测加固中的应用探索 [J]. 土木 建筑工程信息技术 ,2016,8(5):26-29.

[2] 马迅. BIM 技术在既有建筑检测加固中的应用探析 [J]. 安徽建 筑, 2018,24(05):95-96.

[3] 蒋璐. BIM 技术在既有建筑检测中的应用研究 [J]. 土木建筑工程 信息技术 ,2017(3):94-99

[4] 蒲红克, 魏庆朝. BIM 技术在地铁施工过程周边建筑加固中的应 用 [C]. 第十届建构筑物改造和病害处理学术研讨会、第五届工程 质量学术会议论文集.2014.

[5] 张红歌. BIM 技术在既有建筑改造中的应用探究 [D]. 西南交通大 学, 2016

[6] 张俊傥, 李永录. BIM 技术的发展趋势及其在检测鉴定中的应用 研究 $[C]$. 全国工程结构安全检测鉴定与加固修复研讨会 .2015.

[7] 既有建筑抗震加固改造设计项目的风险管理研究 [D]. 中国科学院 大学 (工程管理与信息技术学院),2016.

[8] BIM 技术在既有建筑改造建设期精细化管理中的应用 [D]. 长春工 程学院, 2017 .

[9] 吴建锋. BIM 技术在智能建筑中的应用 [J]. 智能建筑, 2013(10):25 